\title{
Highly flexible strain sensor based on pop up dipole antenna for on-body applications
}

\author{
Shaghayegh Soltani, John C Batchelor \\ University of Kent, Canterbury,UK andss969@kent.ac.uk, J.C.Batchelor@kent.ac.uk
}

Keywords: Strain, Stress, Buckling, Pop up, FEM.

\begin{abstract}
Soft electronic systems are intensively desirable due to their similar mechanical properties to human skin. Technological advances in flexible skin-attachable strain sensors over the last decade have made them attractive for medical applications, in particular, out-of-the-clinic health monitoring. Here we report a novel highly stretchable strain sensor based on a compressive buckling technique for on-body applications. Our suggested sensor is able to measure 0 to $60 \%$ strain with the frequency shift in the range of $2.4 \mathrm{GHz}$ to $5 \mathrm{GHz}$ while keeping the onskin antenna gain greater than $-11 \mathrm{dBi}$. Finite Element Analysis (FEA) using COMSOL Multiphysics ${ }^{\circledR}$ 5.3a was adopted to analyse the buckling behaviour of the $2 \mathrm{D}$ metallic precursor as well as the antenna performance. Numerical results are in good agreement with the experimental data.
\end{abstract}

\section{Introduction}

Flexible sensors offer the capability of integrating with the human body. These devices are mountable on different curvilinear surfaces, which makes them ideal candidates to be placed directly onto the skin. Designing an efficient sensor capable of detecting the amount of skin strain as well as providing real time sensing data is of great interest.

To date several attempts have been made to integrate antenna design for measuring the amount of strain [1-5]. For example changes to the length and width of a patch antenna due to the applied strain in the range of $(0 \%-2 \%)$ resulted in a resonant frequency down-shift between $5.8 \mathrm{GHz}$ to $5.7 \mathrm{GHz}$ [1] .Other study has reported an antenna design based on flexible multilayered graphene film which can detect tensile and compressive bending with strain sensitivity of 9.8 and 9.36 , respectively [2]. Strain sensitivity relations can be found in [3].

Furthermore, Kanaparthi et al. [3], have worked on a rectangular patch antenna on paper substrate with the strain sensitivity of 3.23 and 3.34 to $1.67 \%$ of compressive and tensile bending strain, respectively.

This paper aims to contribute to this growing area of research by incorporating compressive buckling technique to the antenna design. Compressive buckling technique which first suggested by Xu et al. [6], is an efficient way of transforming 2-dimensional (2D) structures to the $3 \mathrm{D}$ ones by using a very simple and cost effective fabrication method. This method is similar to those found in pop up books. So far only the mechanical aspect of this technique has been studied and to the best of our knowledge no previous study has investigated the effect of compressive buckling on the antenna performance.

This work is divided in to two steps. At first nonlinear buckling behaviour of a ribbon made of metal under different amounts of strain was studied and then results from the first step were coupled to the radio frequency physics in order to measure the antenna performance.

\section{Methods}

The proposed strain sensor function is based on the resonant frequency shift of a half wavelength dipole antenna made of 30 $\mu \mathrm{m}$ thick copper. Fig. 1 shows the schematic of the dipole and all dimensions parameters are mentioned in Table 1. The antenna has been designed to operate at $2.4 \mathrm{GHz}$ without any strain on an ultra-thin, elastomeric Silicone rubber substrate ( $0.4 \mathrm{~mm}$ thickness) with the commercial name of Dragon Skin which has effective elastic modulus $\left(\mathrm{E}_{\text {Substrate }}=166 \mathrm{kPa}\right)$ and Poisson ratio $(v$ substrate $=0.49)$ similar to human skin $[6]$.

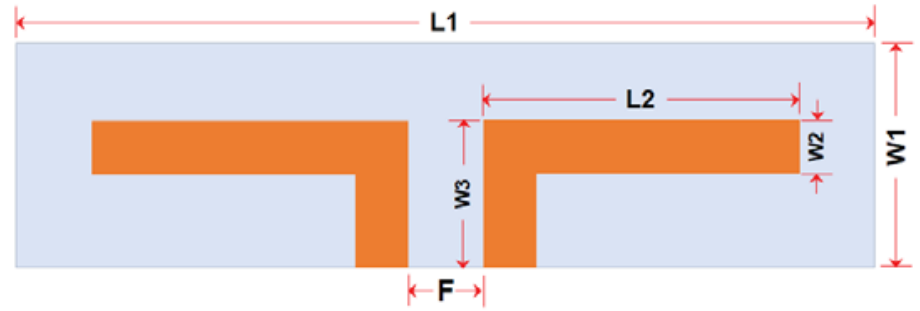

Figure1: Schematic of half wavelength dipole antenna.

Table 1. Dimension parameters of the antenna design

\begin{tabular}{lcccccc}
\hline Parameters & L1 & L2 & W1 & W2 & W3 & F \\
Dimensions (mm) & 40 & 16 & 10 & 1 & 5.5 & 2 \\
\hline
\end{tabular}

Structural mechanics module of COMSOL Multiphysics ${ }^{\circledR}$ software was used to model the antenna deformation with $0 \%$ to $60 \%$ strain.

Fig. 2 illustrates antenna samples for 0 to $60 \%$ strain as well as their functions on human forearm. 


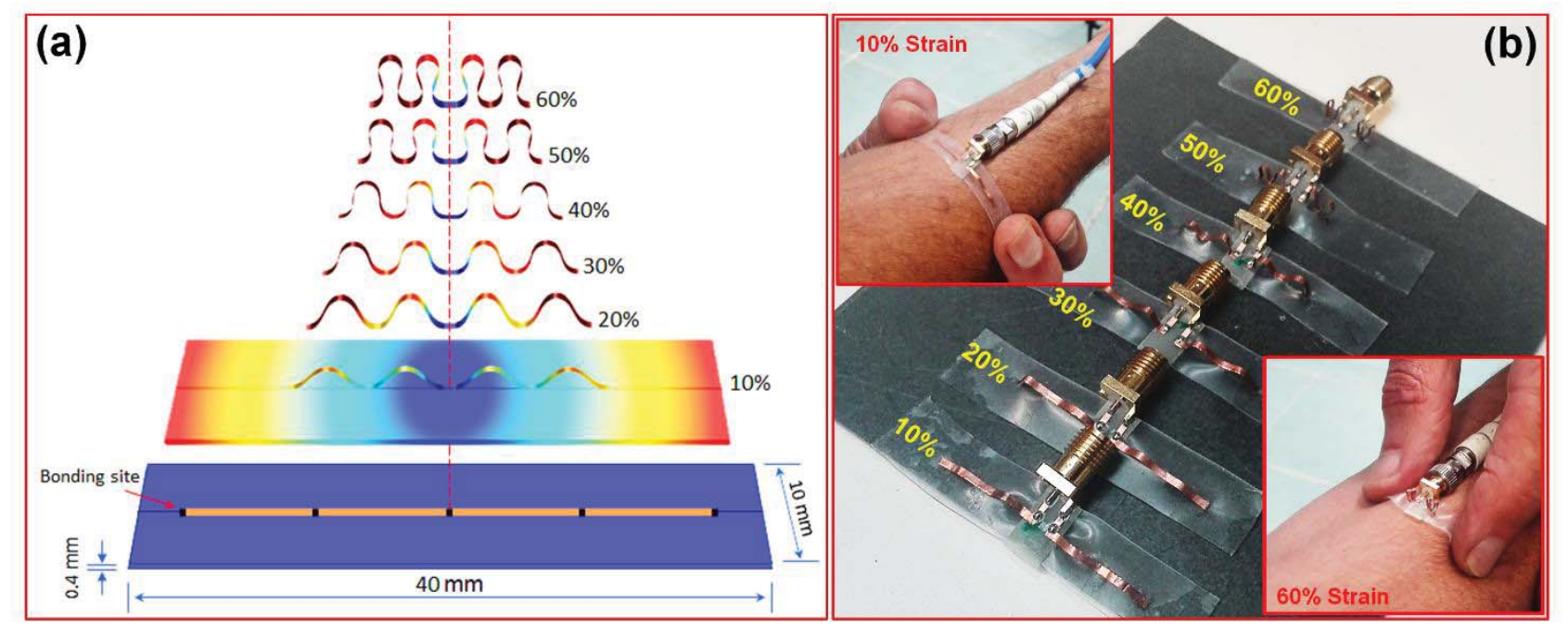

Figure 2: Buckled ribbon with different uniaxial strains on the elastomeric substrate. a) Simulation and b) Antenna samples, the antenna was tested on forearm.

Mooney Rivlin model (two parameters) was utilised to model the hyperelastic substrate, which can fit the measured nonlinear stress-strain curves of isotropic rubber-like materials very well [9].

$$
\Psi=\sum_{i=1}^{2} C_{i}\left(I_{i}-3\right)
$$

Where $\Psi$ is strain energy function, $\mathrm{I}_{1}$ and $\mathrm{I}_{2}$ are Cauchy- Green invariants, $\mathrm{C}_{1}$ and $\mathrm{C}_{2}$ are Monney Rivilin parameters.

After post-buckling analysis, the deformed structure was coupled to the RF module for evaluation of its electromagnetics characteristics as a half wavelength dipole antenna. The antenna was placed on forearm which was modelled with a multi-layered cubic structure with the dimension of $60 \mathrm{~mm} \times 60 \mathrm{~mm} \times 20 \mathrm{~mm}$ consisting of bone, muscle, skin and fat with their specific permittivity and conductivity in the frequency range of interest $[8,11]$. The electrical properties of the elastomeric substrate was measured as $1.6 \pm 0.2$ and $0.04(\mathrm{~S} / \mathrm{m})$ for permittivity and conductivity, respectively. The input impedance of antenna is $50 \Omega$ and perfectly matched layer (PML) boundary condition was used for absorption of outgoing waves. As the accuracy of solution intensively depends on the mesh size, mesh refinement was adopted to minimise the error between the real and approximated solutions.

\section{Results and Discussion}

Skin strain due to the human motion causes a tensile stress in the substrate and when the skin returns to its normal condition the substrate is released to its original size. As a result of this stretch and release mechanism, a compressive stress is induced into the dipole antenna with the selective bonding to the substrate and initiates nonlinear buckling process which cause an extension to the third dimension. Fig. 3 represents the Von

mises stress $(\mathrm{MPa})$ along with both the substrate and the metallic ribbon with $10 \%$ and $60 \%$ strain, respectively.

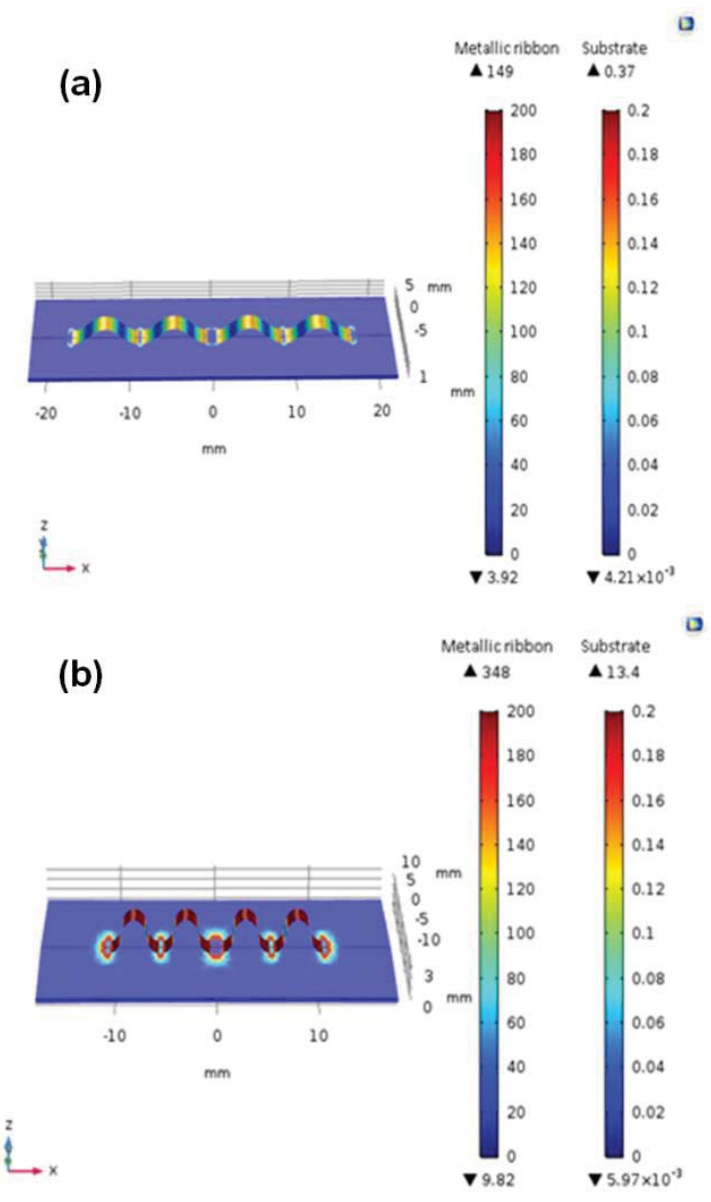

Figure 3: Von mises stress for a) $10 \%$, and b) $60 \%$ strain.

From the Fig. 3 it can be seen that amount of stress close to the bonding sites as well as the bend apexes is much higher than other parts. 
Due to the out of plane displacement during the buckling, a mutual capacitance occurs between the dipole adjacent arms which leads to a decrease in the overall capacitance. This factor along with the existence of air gaps between the substrate and the buckled sections will result in a quite large resonant frequency shift. By increasing the strain level, distance between two arms in each meander decreases while the height of each bend becomes greater. As the arms get closer, an increase of the in-series combined mutual capacitance is expected [12]. A decrease in the overall capacitance as well as existence of larger air gap between the dipole and substrate lead to a larger frequency shift.

Fig. 4 plots reflection coefficient $\left(S_{11}\right)$ versus frequency both for numerical and experimental data. Numerical data illustrates that when dipole antenna is in flat mode ( $0 \%$ strain) it works at $2.4 \mathrm{GHz}$ and when it goes under the maximum compressive strain $(60 \%)$ it works at $5 \mathrm{GHz}$. Note, the antenna $\mathrm{S}_{11}$ remains better than $-20 \mathrm{~dB}$ across the entire stretch range.

Experimental measurement represents a maximum frequency tolerance of almost $5 \%$ which is due to the fabrication accuracy.

Fig. 5 presents the frequencies related to the strain values between $0 \%$ and $60 \%$ which shows a nonlinear behaviour. It suggests that there is a significant frequency up-shift of about $800 \mathrm{MHz}$ from $0 \%$ to $10 \%$ substrate strain and it reduces further to $500 \mathrm{MHz}$ and $400 \mathrm{MHz}$ for $20 \%$ and $30 \%$ strain, respectively, and then remains constant at about $300 \mathrm{MHz}$ for further strain percentages.

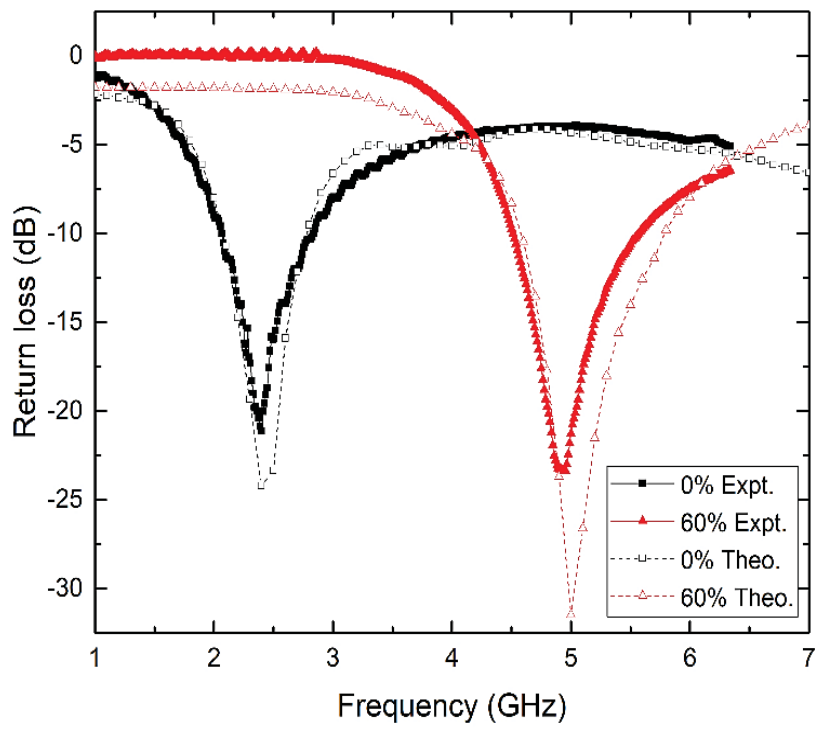

Figure 4: Numerical and experimental reflection coefficient $(\mathrm{dB})$ of half wavelength dipole antenna versus frequency $(\mathrm{GHz})$ with $0 \%$ and $60 \%$ strain.

Fig. 6 shows the E-Plane radiation pattern of the antenna with $0 \%$ and $60 \%$ strain. The antenna has a gain of greater than almost $-11 \mathrm{dBi}$ while keeping on skin which is expected due to the loss within human tissues.

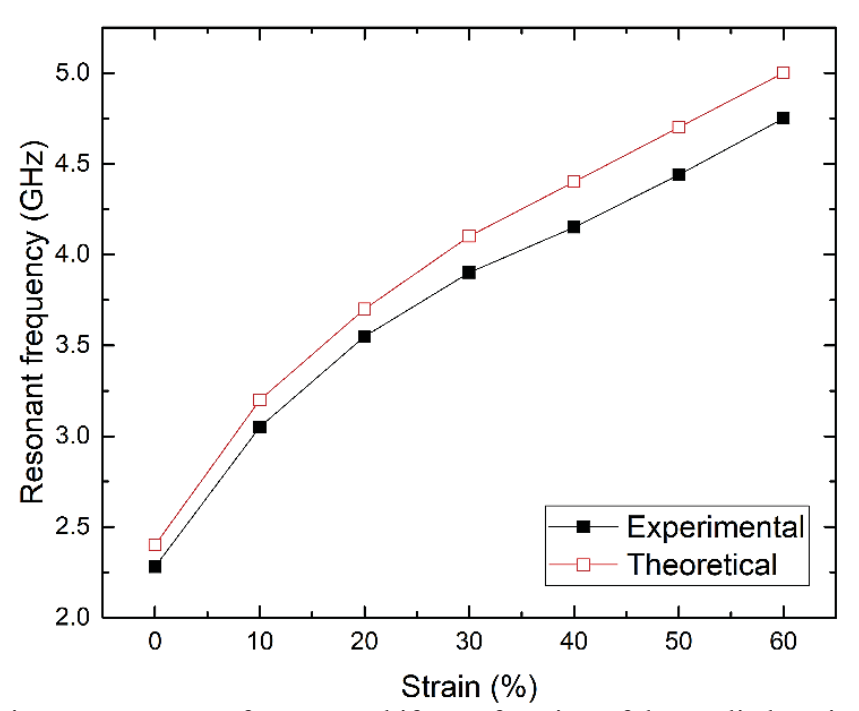

Figure 5: Resonant frequency shift as a function of the applied strain.
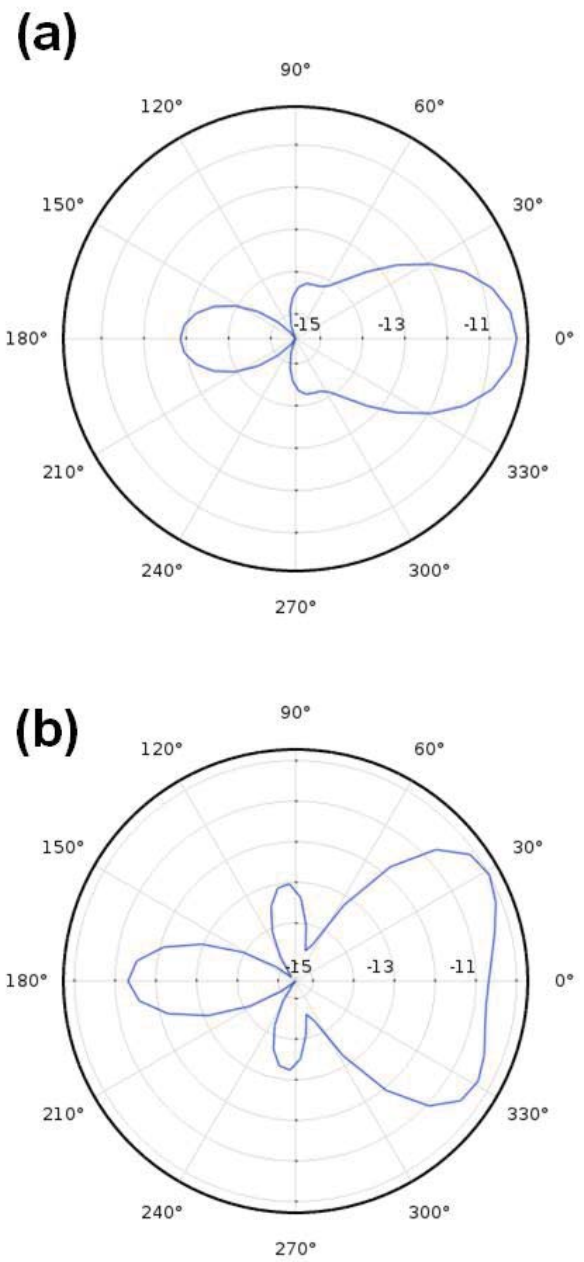

Figure 6: E-Plane Far field gain (dBi) for, a) $0 \%$, and b) $60 \%$ strain.

\section{Conclusion}

This paper presents the design, simulation result and fabrication of a novel flexible strain sensor for body mounting 
applications. This sensor works based on resonant frequency shift of a half wavelength dipole antenna which is deformed according to a compressive buckling technique. The proposed sensor is capable of detecting up to $60 \%$ strain with significant frequency shift in the range of $2.4 \mathrm{GHz}$ to $5 \mathrm{GHz}$. The antenna gain is greater than $-11 \mathrm{dBi}$ when directly attached to skin for the whole strain range of interest. Antenna samples were tested on body and there was a good match between numerical data and experimental measurements.

\section{Acknowledgements}

This research was supported by the UK Engineering and Physical Science Research Council (EPSRC). We are thankful to our colleague Simon Jakes for helping us in fabrication and measurement.

\section{References}

[1] B. Abderrahmane, S. Abdulkadir, Z. Ralf, M. Christian, K. Olfa. "Flexible Strain Sensor based Microstrip Patch Antenna", 15th International Symposium on Microwave and Optical Technology, Dresden, Germany, (2015).

[2] D. Tang, Q. Wang, Z. Wang, Q. Liu, B. Zhang, D. He, Z. $\mathrm{Wu}, \mathrm{S}$. Mu, "Highly sensitive wearable sensor based on a flexible multi-layer graphene film antenna", Science Bulletin, 63, pp. 574-579, (2018).

[3] S. Kanaparthi, V. R. Sekhar, S. Badhulika. "Flexible echofriendly and highly sensitive paper antenna based electromechanical sensor for wireless human motion detection and structural health monitoring", Extreme Mechanics Letters, 9, pp. 324-330, (2016).

[4] U. Tata, H. Huang, R. L. Carter, J. C. Chiao. "Exploiting a patch antenna for strain measurements", Measurement Science and Technology, 20, pp. 1-7, (2008).

[5] X. Chen, L. Ukkonen, T. Björninen. "Passive E-Textile UHF RFID-Based Wireless Strain Sensors with Integrated References", IEEE Sensors Journal, 16, pp. 7835-7836, (2016).

[6] S. Xu, Z. Yan, K. I. Jang, W. Huang, H. Fu, J. Kim, Z. Wei, et al. "Assembly of micro/nanomaterials into complex, three dimensional architectures by compressive buckling", Science, 347, pp. 154-159, (2015).

[7] D. H. Kim, N. Lu. R. Ma, Y. S. Kim, R. H. Kim, S. Wang, J. Wu, S. M. Won, H. Tao, et al. "Epidermal Electronics", Science, 333, pp. 838-843, (2011).

[8] M. A. Ziai, J. C. Batchelor. "Temporary on-skin passive UHF-RFID transfer tag", IEEE transactions on antennas and propagation, 59, pp. 3565-3571, (2011).

[9] P. Martins, R. N. Jorge, A. Ferreira. "A comparative study of several material models for prediction of hyperelastic properties: Application to Silicone-Rubber and Soft Tissues". Strain. 42, pp. 135-147, (2006).

[10] COMSOL multiphysics ${ }^{\circledR}$ v. 5.3a. www.comsol.com. COMSOL AB, Stockholm, Sweden.

[11] Tissue properties database | IT IS Foundation [online], https://itis.swiss/virtual-population/tissueproperties/database/
[12] O. O. Olaode, 'Research and Development of LowProfile, Small-Footprint Antennas for VHF-UHF range of Applications', $\mathrm{PhD}$ dissertation, Duke University, (2004). 